\title{
DE LA FRAYE DE LA CARPE, DU ROTENGLE ET DE LA TANCHE A L'ÉTABLISSEMENT DOMANIAL DE CYPRINICULTURE DE COURVILLE PRÈS DE FISMES (MARNE) ET DANS LES ETANGS ET CANAUX DU DOMAINE NATIONAL DE RAMBOLILLET

\author{
par M. RENÉ CHARPY \\ Inspecteur des Eaux et Forêts \\ Chel de la $1^{\text {ro }}$ Région Piscicole
}

L'établissement de cypriniculture de Courville, près de Fismes, dans la Marne, a été acquis par l'Etat (Administration des Eaux et Forêts) en Mars 1937. Ce domaine comporte 13 étangs d'une superficie tolale de 8 hectares 10 ares, à savoir : -7 ares, -30 ares, -3 ares, -1 hectare 90 ares, 45 ares, - 63 ares, -1 hectare 70 ares, -80 ares, -5 ares, -5 ares, - 5 ares - et 1 hectare 90 ares.

Dans une conférence que nous avons faite le Samedi 23 Octobre 1943, devant la Sociélé des Amis du Muséum National d'Histoire Naturelle, à Paris, nous avons exposé les principes et les résultats de la gestion de cet établissement, gestion que nous assurons depuis sept ans.

Nous donnons ci-après un extrait de cette conférence pour ce qui $\mathbf{s}^{+} \mathbf{y}$ rapporte à la fraye de la Carpe, du Rotengle et de la Tanche.

\section{MISE EN EAU DES ÉTANGS}

A Courville, tous nos étangs sont en eau au $1^{\text {er }}$ Avril environ, de telle sorte que nos géniteurs Gardons et Tanches puissent étre mis en place dans la première quinzaine ou tout au moins au début de la deuxième quinzaine du même mois. Pour ce faire, on commence à alimenter les étangs en eau dès le 15 Mars.

(1) Note extraite d'une conférence faite par l'auteur, le 23 Octobre 1943, à la Societé des Amis du Museum National d'Histoire Naturelle. 
Nous ne remplissons pas les étangs brutalement mais au contraire progressivement, et au fur et à mesure de la poussée de la végétation de façon à ce que celle-ci soit aussi abondante que possible. Nous pensons, en effet, - comme MM. Duche, Lefève et Spillmaxn (1), membres de la Commission de Pisciculture du Commissariat de la Sologne - "qu'une certaine végétation phanérogamique doit être laissée dans les étangs d'alevinage jusqu'à la fin de Juillet pour servir de frayères aux géniteurs et de refuge aux jeunes allevins $"$.

De là, faut-il aller à dire, comme les auteurs précités, qu'il convienne dans les étangs à Carpes, de faire disparaître délibérément la végétation submergée parce que celle-ci absorberait des sels nutritifs au détriment du plancton? Nous ne pensons pas qu'il faille être aussi catégorique et nous croyons que ce qui vaut peut-être pour les étangs de la Sologne, ne saurait être généralisé a priori. Au surplus, si la végétation submergée absorbe des sels nutritifs dont aurait pu profiter le plancton, et par conséquent le poisson, par contre, elle présente certains avantages tels que de fournir à l'eau de l'oxygène et d'abriter une faune particulière qui peut suppléer, dans une certaine mesure, à là rareté momentanée de plancton. Il n'y a d'ailleurs pas que le plancton; il y a aussi la faune de fond à laquelle les auteurs précités n'attachent pas, semble-t-il, assez d'importance; il faut tenir compte enfin de ce que certains étangs ne sont pas " naturellement " riches en plancton et de ce que, par suite, la faune abritée par la végétation submergée y revêt une importance toute spéciale.

Aussi, quitte à modifier ultérieurement notre point de vue, tant que des expériences méthodiques et concluantes n'auront pas été réalisées à ce sujet, nous ne nous hasarderons pas, pour notre part, à supprimer délibérément la végétation submergée dans les étangs à Carpes. Nous éviferons seulement, par des faucardages, qu'elle ne devienne envahissante.

\section{MISE EN PLACE DES GÉNITEURS}

On pourrait envisager de consacrer tel étang à la production de l"alevin de Carpe, tel autre à l'alevin de Gardon, etc., mais en fait, étant donné que, suivant les années, la Carpe ou lè Gardon ou la Tanche alevine plus ou moins bien, il paraît rationnel de prévoir dans les étangs d'alevinage à Cyprins, à la fois des géniteurs de ces trois espèces afin d'être à peu près sur que l'une d'elles au moins donnera des produits de quelque importance. Au surplus, la Tanche, utilisant le fond, n'entravera en rien l'élevage des espèces de surface et de demi-fond.

En principe, les géniteurs sont mis à l'eau lorsque la température de cette eau atteint $19^{\circ} \mathrm{C}$. environ ; pratiquement, ils sont déversés dans les étangs de pose vers le 15 Avril. Pour le Gardon, facilement blessé pendant les pêches, il est à conseiller de n'en faire le reprise dans l'étang d'hiver-

(1) Action Forestière et Piscicole. Juin 1943, na 75. 
nage que peu avant la fraye, pour ne pas donner le temps, aux blessures, d'évoluer avant cette époque, jusqu'à la mort du poisson. La Tanche, de même que la Carpe, est moins délicate.

Pour la Carpe, nous prenons soin de conserver un petit lot de géniteurs en eau froide pour n'ètre déversé en étang qu'à une date ultérieure (10 Juin environ), en vue de parer à la déficience éventuelle des premières frayes. Nous envisageons même de conserver tous nos géniteurs Carpes en eau froide jusqu'au 10 Juin, afin d'éviter que la fraye des poissons de cette espèce ne se produise trop tôt dans l'année et ne soit annihilée, ou tout au moins partiellement détruite, par les froids.

Au lieu de faire poser ces géniteurs de réserve en étang, nous utilisons parfois des frayères "Dubish "lorsqu'il est constaté que la ponte directe en étang s'avère défectueuse.

En général, ce n'est pas le cas dans les étangs de Courville, de sorte que les frayères "Dubish " ne constituent là qu'un accessoire non indispensable :- elles présentent certes des avantages dont le principal réside dans l'obtention automatique de la ponte, c'est-à-dire à jour voulu ou presque, si l'on n'y déverse les géniteurs que lorsque l'eau y est suffisamment chaude, - mais aussi des inconvénients : en effet, la présence des géniteurs dans l'espace restreint de la frayère est une cause de pertes dans leur progéniture durant l'incubation des ceufs comme après leur éclosion. Il faut donc enlever les géniteurs dès que possible et pour le faire en toute facilité, on est óbligé de vider partiellement la frayère jusqu'à ce qu'il ne reste plus d'eau que dans la partie la plus creuse où les reproducteurs se réfugient et deviennent faciles à capturer. Durant liopération, une partie du bassin est donc asséchée, ce qui ne manque pas d'exercer une influence néfaste sur les oufs adhérents aux herbes émergées.

M. l'Ingénieur Docteur Boris Kostomanov a entrepris des recherches à ce sujet à l'établissement de Mlada Vozice en Bohëme. Il résulte de ses constatations (1) que, dans l'ensemble, le déchet serait de l'ordre de 40 à $55 \%$; mais si l'on admet que l'abaissement du niveau d'une frayère n'affecte que moitié environ des oufs qui y ont été pondus, le déchet dans une frayère partielleemnt asséchée durant deux heures ne serait que de $20 \%$ environ, chiffre dit M. Kostomarov sans importance, puisqu'une femelle pond normalement 300 à 400.000 ocufs. Pour lui, un déchet du cinquième de cette quantité laisse escompter l'éclosion de 240.000 à 320.000 alevins, ce qui suffit.

Il n'en reste pas moins qu'il faut prendre de grandes précautions pour que le frai ait à pâtir le moins possible de la pèche des géniteurs par assèchement partiel ; qu'il faut agir vite pour qu'entre la vidange de la frayère et sa remise en eau il ne s'écoule pas plus de deux heures environ, et que,

(1) Wie lange koennen befruchtete Karpfeneier ohne Schlüpfungs-Verluste ausser Wasser-bleiben ? - Fischerti Zeitung, Neumann, Neudamm, Tome XLIII. no 42, 1939.

Voir aussi : - Bulletin francais de Piscicullure no 125, Avril-Juin 1942, p. 132. 
par suite, il faut disposer pour l'opération d'ouvriers réellement spécialisés.

Ce n'est pas le seul inconvénient : il faut encore repêcher les alevins pour les déverser en étang, et ce dès qu'ils ont résorbé leur vésicule ombilicale ; or, à cette époque, il fait généralement très chaud, de sorte que mẻme en procédant de bonne heure le matip, il se produit un déchet assez appréciable sur les alevins : il faut alors prendre soin de placer les bidons dans l'eau mème des fossés et, sans sortir les alevins de l'eau, de les faire passer dans les bidons à l'aide de petits troubles garnis de tulle fin ou de linge.

Pour toutes ces raisons, nous préférons, lorsque les étangs conviennent à la pose, ne pas utiliser les frayères "Dubish".

Nous avons également utilisé ces frayères pour le Gardon avec des résultats appréciables, mais il faut noter qu'une frayère de 60 mètres carrés seulement constitue pour les Hérons une véritable tentation et que, en quelques instants, tous les géniteurs d'une frayère à Gardons peuvent ètre " liquidés " par les Hérons.

De sa très intéressante étude algologique sur le domaine national piscicole de Rambouillet (1), M. Marcel Lefève, Assistant de Cryptogamie au Muséum National d'Histoire Nalurelle, a cru pouvoir aboulir à des conclusions pour l'aménagement piscicole des plans d'eau considérés, notamment en ce qui concerne le nombre de géniteurs à cmployer. Nous nous permettrons de discuter le bien-fondé de certaines d'entre elles.

En particulier, M. LefÈva préconise pour le peuplement de l'étang du Rondeau, l'emploi de 100 reproducteurs Carpes sélectionnées et d'autant de Gardons, ce qui - le Rondeau ayant une superficie de 9.000 mètres carrés - représente à l'hectare 111 reproducteurs Carpes et 111 reproducteurs Gardons. Or, si le nombre de reproducteurs Gardons ne nous semble pas exagéré, celui des Carpes paraît beaucoup trop élevé ; à Courville, nous en prévoyons 16 fois moins, soit 7 à l'hectare au lieu de 111 , à raison de. 3 femelles pour 4 mâles. Nous ne prétendons pas que notre chiffre ne puisse être modifié et ce n'est d'ailleurs là qu'un chiftre moyen, mais nous pensons qu'en augmentant notablement le nombre de reproducteurs Carpes, on n'obtiendrait pas plus d'aleving et même pas du tout si l'on exagérait : le surpeuplement n'a pas seulement des conséquences néfastes du fait que le nombre des "mangeurs " n'est pas proportionné à la quantité de nourriture, c'est-à-dire à la capacité biogénique, mais aussi du fait que la Carpe, étant particulièrement vorace, détruit un grand nombre d'ceufs et d'alevins, même de son espèce. L'alevinage de la Carpe ne peut donc réussir dans un plan d'eau considéré si la charge en Carpes adultes y est par trop forte, et de même, l'alevinage du Gardon en pâtit. C'est, peut-être, une des raisons pour laquelle l'étang du Rondeau renfermait entre autres, en Mars 1940, aux dires mêmes de M. Lefevre, 1.500

(1) Voir :- Bulletin franqais de Pisciculture, no 122, Juillet 1940 - Juln 1941, pp. 89 et ss. 
Carpes de 2 à 3 livres et seulement quelques dizaines d'alevins de Carpes d'un été et aucun alevin de Gardon.

Dans les grands étangs de Courville, nous employons donc 7 géniteurs Carpes à l'hectare ( 3 femelles et 4 mâles). Nous y adjoignons 25 Tanches et 150 Hotengles à l'hectare.

Pour les Carpes, il semble, d'après les constatations que nous avons pu faire dans les frayères "Dubish ", que l'on obtienne de meilleurs résultats en opérant avec des mâles de dimensions variées, des petits, des moyens et des gros.

Pour les Tanches, nous mettons autant de mâles que de femelles, mais peut-être conviendrait-il mieux, comme le conseille Wunder (1), d'employer deux mâles pour une femelle.

Le chiffre de 150 Rotengles à l'hectare peut paraître trop élevé et est peut-être trop élevé, mais il faut considérer qu'il est pratiquement impossible, pour l'ouvrier pisciculteur, du moins avant l'epoque de la fraye, de reconnattre les mâles des femelles de sorte que l'on est obligé de forcer un peu pour être à peu près assuré d'avoir le nombre de femelles voulu. Avec les Gardons blancs, dont les mâles se distinguent assez aisément des femelles, i] serait possible de réaliser un peuplement beaucoup plus rationnel.

\section{LA FRAXE}

Il est classique de dire que le Rotengle pond en Avril-Mai, la Carpe au début de l'été et la Tanche de Mai en Juillet.

A Courville, sauf quelques exceptions, la Carpe pond en fait très généralement avant les deux autres especes. D'autre part, ni pour la Carpe, ni pour le Rotengle, ni pour la Tanche, il n'a été constaté de fraye en Avril du moins depuis 1932, époque à laquelle remonte notre documentation sur la question.

Il semble que pour la Carpe, la fraye dépende assez étroitement de la température de l'eau : la fraye de la Carpe s est en effet toujours produite, depuis 1932 , lorsque la température de l'eau atteignait $18^{\circ}, 18^{\circ} 5$ ou $19^{\circ}$.

$\mathrm{Si}$, d'ailleurs, l'on attend pour déposer les géniteurs Carpes dans les frayères "Dubish " que la température de I'eau y ait atteint 19 a $20^{\circ}$, le déclenchement de la ponte y est quasi automatique.

Bien entendu, la date même de la fraye de la Carpe dans les étangs de Courville étant fonction de la température, varie suivant les années; et dans la mème année, suivant les étangs. De 1932 à 1943 , les frayes de la Carpe se sont ainsi élagées entre le 3 Mai en 1943 (étang 4) et le 10 juin en 1935 (même étang 4) : en règle très générale, cette fraye se produit du 14 au $29 \mathrm{Mai}$; en outre, dans un même étang, il ne s'écoule jamais un très grand laps de temps entre les frayes successives de la mème année.

(1) Beobachtungen und Vorschlaege zur Schleienzucht in der Karpfenteichwirtschaft. - Allegemeine Fischerei Zeilung. Angsbourg, no 15, 1er Aout 1938, p. 225. 
En 1934, par exemple, les Carpes ont frayé dans l'étang 4, les 3 et 14 mai ; - en 1935 , les $26 \mathrm{Mai}$ et 10 Juin. La même année, il y a cependant parlois des différences assez notables entre les dates des frayes de la Carpe suivant les étangs, ceux-ci n'étant pas tous placés dans des conditions identiques: Si, par exemple, en 1942, la Carpe a fravé le 24 Mai dans les étangs 4 et 11 et le 26 Mai dans les étangs 5 et 6 ; et si en 1941 la fraye s'est produite le 28 Mai dans l'étang 4 et le 29 Mai dans l'étang 11 , en 1934 les Carpes ont frayé le 3 Mai dans l'étang 4, les 12 et 13 Mai dans l'étang 7 , le 14 Mai à nowveau dans l'étang 4, le 25 Mai dans l'étang 7 et le 31 Mai dans l'étang 6.

La fraye du Gardon rouge ou Rotengle ne paraft pas être en aussi stricte relation avec la température de l'eau que la fraye de la Carpe. Nous avons eu l'occasion, en effet, de noter des fraves de Rotengle dans des eaux marquant $18,19,20,21,23,24,25,26$ et $27^{\circ}$. En outre, alors que dans une station déterminée tous les Gardons blancs pondent au même moment, la ponte du Gardon rouge ou Rotengle se prolonge sur une certaine période et il y a une série de frayes successives séparées parfois par des laps de temps assez appréciables; on constate donc, dans les étangs à Rotengle, Ja présence d'alevins de plusieurs dimensions correspondant à ces diverses frayes successives.

En 1941, par exemple, dans l'étang 4, les Gardons ont frayé les 6 Juin, 5 Juillet et 18 Juillet; en 1942 et dans le mème étang, les 6 Juin et et 20 Juillet; en 1943, toujours dans I'étang 4, les 20 Mai, 3 Juin et 10 Juin.

De 1932 à 1943, les frayes des Rotengles se sont étagées entre le 3 Mai dans l'étang 4 en 1933 et le 25 Juillet dans les étangs 5 et 6 en 1942 .

Pour la Carpe, il est possible de parler d'une période movenne de fraye (14-29 Mai) ; pour le Rotengle, il existe plusieurs périodes de fraye dont Ies limites se déplacent chaque année en fonction de divers facteurs, dont la température de l'eau.

La fraye de la Tanche, comme celle du Rotengle ne paraît pas dépendre intimement de la température de l'eau : nous avons constaté des frayes de Tanche dans des eaux marquant $18^{\circ}, 23^{\circ}, 24^{\circ}, 25^{\circ}$ et $26^{\circ} \mathrm{C}$.

La Tanche, d'autre part, peut frayer parfois à une température d'eau $\left(18^{\circ}\right)$ moins élevée que la Carpe, de sorte que, dans les étangs relativement frais, la Tanche peut aleviner certaines années alors que la Carpe ne s'y reproduit pas.

De 1932 à 1943, les frayes des Tanches se sont étagées entre le 24 Mai en 1934 (étang 8) et le $1^{\text {or }}$ Aont en 1941 (étangs 5 et 11). A Courville, la la Tanche pond généralement en Juin et Juillet et, plus spécialement, fin Juin - début de Juillet.

Si la Carpe fraye le plus souvent sur les plantes des berges, elle fraye parfois en pleine eau sur les Potamots. Il en est de même de la Tanche : le Potamot à feuilles flottantes notamment convient bien à la Tanche qui 
y dépose fréquemment ses ceufs, ce qui a fait donner à cette plante le nom d'herbe à Tanche.

Quant aux Rotengles, nous n'en avons jamais constaté de fraye en pleine eau, mais toujours sur les plantes des berges, plus précisément sur les racines et radicelles des graminées, cypéracées, etc... qui croissent sur les berges. Tout au plus avons-nous pu noter, ailleurs qu'à Courville, et ce bien rarement, des frayes de Rotengle sur des herbes flottantes à proximité même des berges. Si donc, avec juste raison semble-t-il, M. Marcel LFFł̀re, dans l'article que nous avons déjả cjté, a préconisé l'introduction dans l'étang du Rondeau (domaine de Rambouillet) de certaines plantes aquatiques telles que Ies Ceratophyllum, certains Myriophyllum et Potamogeton, nous ne pensons pas qu'il faille, comme lui, affirmer - étant donné que dans le Rondeau on cherche surtout à développer le Rotengle que cette introduction " résoudrait à la fois le problème des frayères, des refuges pour les alevins et de l'alimentation plus rationnelle du Poisson ". Il eut été utile de préciser que cet apport de végétation phanérogamique, tout en étant très précieux pour favoriser la fraye de la Carpe, ne saurait avoir aucune action directe sur la fraye du Rotengle qui lui, ne parait jamais pondre en pleine eau : les berges du Rondeau sont retenues sur tout le pourtour par un épais mur de pierres ; il y a donc peu de chance pour que la fraye du Gardon puisse y être sensiblement améliorée, à moins que I'on $y$ utilise des frayères artificielles et que ces frayères soient établies en tenant compte des données mêmes de la nature.

M. Marcel Lefèvne, il est vrai, avait conseillé l'emploi de frayères artificielles dans le Rondeau, en attendant que la végétation phanérogamique y soit suffisamment développée.

Dans les Canaux du Domaine de Rambouillet, la température de l'eau, au cours du mois de Juin, est nettement suffisante pour permettre la fraye de la Carpe et, cependant, celle-ci ne s'y reproduit pas.

En 1941, M. Lefèvre y a suivi le comportement des Carpes à l'époque de la fraye : - le 4 Juin, la température de leau y était de $24^{\circ} \mathrm{C}$, , " l'activité des Carpes était extraordinaire. Dans tous les endroits de hauts fonds, elles étaient rassemblées par centaines, se poursuivant sans arrêt, se frotlant contre les berges, et donnaient I'impression d'une activité sexuelle intense.

"Cet état ne dura que deux journées.

"Malheureusement, tous Ies individus capturés par la suite furent trouvés porteurs de laitance ou d'œufs non expulsés; il y a donc apparence de fraye, mais pas fraye effective, au moins pour la grande majorité des individus."

Pour expliquer ce phénomène, M. Marcel Lefìvre a émis l'hypothèse de l'influence d'une trop forte proportion de tannin dans l'eau, en provenance de la chute annuelle des feuilles d'arbres.

Il est possible qu'en cela $M$. Marcel Lefève ait raison et que, comme 
l'a écrit Guenaux (1), il convienne, pour la reproduction de la Carpe, d'éviter les étangs de bois "à cause du tannin dont les eaux seraient chargées ".

Le fait toutefois n est nullement prouvé : il faudrait pour cela des expériences directes d'action du tannin sur le frai de la Carpe, et, d'autre part, il se pourrait que le phénomène décrit par M. LF.Fève puisse g'expliquer d'autre manière : les Canaux du Domaine de Rambouillet sont en effet totalement dépourvus de végétation phanérogamique; par suite, la Carpe dont les oufs et la laitance paraissent arriver à maturité, ne trouve dans ces plans d'eau aucun support pour y déposer ses ceufs, ou plutôt pour aggrutiner ses chapelets d'oufs; on pourrait donc supposer qu'elle s'épuise en vain et que les coufs n'étant pas évacués, la fécondation ne puisse pas se produire : pour juger de la cause, il suffirait d'ótablir, en des emplacements judicieusement choisis, des frayères artificielles à claies ou à fascines par exemple.

Dans le Rondeau, également dépovrvu de vegétation phanérogamique, la Carpe ne dispose pour frayer que des racines immergées des Cyprès chauves et des détritus accumulés dans les angles de la pièce d'eau. Or, la fraye de la Carpe n'y est jamais que très peu importante, bien que les eaux ne renferment que peu de tannin, les arbres étant assez éloignés du bord de l'eau pour que les feuilles ne tombent pas directement dans l'étang.

De même, des Carpes de belle qualité qui, en attendant leur affectation définitive, avaient été entreposées au Centre d'Hydrobiologie du Paraclet (Somme), dans un bassin restreint, à peu près totalement dépourvu de végétation, n'ont pas frayé.

Pour concrétiser l'influence néfaste qu'aurait le tannin sur la fraye de la Carpe, M. Marcel Lfrève a cité le cas de la Mare des Bécasseaux, très polluée par les feuilles de chêne, où sept Brochets s'y sont fort bien reproduits alors que trente Carpes de trois à quatre étés $n^{\prime} y$ ont donné aucun alevin ; mais, outre que la Carpe ne reproduit qu'à partir de la quatrième année, la quantité de reproducteurs Carpes introduits dans cette mare (qui n"a que quelques ares) est beaucoup trop élevée pour que l'alevinage de la Carpe ait quelque chance d'y réussir. Au surplus, eette mare est dépourvue de végétation phanérogamique.

En somme, si la reproduction de la Carpe est minime dans le Rondeau, si elle est nulle dans les canaux, de même que dans les étangs de bois et mares alimentés par des eaux de fossés lavant des feuilles de chênes (Etang d'Or, étangs de Gruyer, de Coupe-Gorge, du Moulinet, de la Grenouillère, etc...) il serait possible qu'il faille en attribuer la cause. tout au moins en partie, non pas a une action chimique directe des tannins sur la reproduction proprement dite de la Carpe, mais peut-être à la déficience (Rondeau), ou à l'absence (canaux, étangs de bois) de la végétation phanérogamique susceptible de servir de support pour les oufs, déficience ou absence

(1) G. Guenaux, - Piscicullure, - Paris, Baillière, 1923, p. 154. 
provoquée par les conditions hydrobiologiques propres à ces plans d'eau (accumulation de feuilles et de détritus sur le fond, action stérilisante des tannins, fermentations, etc...).

Toutefois, les canaux du Domaine de Rambouillet se déversent dans de petits cours d'eau appelés rivières anglaises, alimentés uniquement par les eaux desdits canaux, donc assez riches en tannin; or, bien que les conditions biologiques de ces rivières anglaises soient très variées (profondeur, éclairement, etc...) et qu'elles soient pourvues en particulier d'une végélation phanérogamique parfois très denss, la Carpe ne šy reproduit pas : il y a seulement apparence de frave sans résultat.

1l y a là un fait qui parait corroborer nettement l'hypothèse émise par M. LeFÈve au sujet de l'influence des tannins sur la reproduction de la Carpe, hyppothèse qui d'ailleurs, nous le répétons, peut correspondre à lia réalité.

Il convient, cependant, de noler que la plupart des bras de ces petits cours d'eau sont, comme le précise M. LeFìvre lui-même "encombrés d'une masse énorme de détritus organiques, branchages et feuilles qui sont le siège de fermentations estivales nauséabondes et très préjudiciables à la gent aquatique ". La. vase ainsi formée "d'un noir d'encre, est surwut recouverte de Cyanophytes filamenteuses qui lui communiquent une odeur caractéristique ".

Dans d'autres bras, il est vrai, la végétation aquatique dense, provoque une épuration de l'eau avec absorption probable des matières organiques dissoutes, de sorte que l'eau y est parfaitement claire et absolument dépourvue de Cyanophytes, mais l'absorption d'acide carbonique consécutive a la fonction chlonophylienne y est telle que le $p H$ peut y atteindre le chiffre énorme de 11 au début de l'été, pour une température de l'eau de $26^{\circ} \mathrm{C}$.

Certes, tout aquiculteur sait depuis longtemps déjà que le $p H$ d'une eau est extrêmement variable au cours de l'année et au cours même d'une journée, suivant les points de prélèvement, l'heure de la journée, la température, l'insolation, la saison, etc...

II n'est pas douteux qu'il y a eu un emballement exagéré pour cette notion de $p H$, commé pour d'autres notions, que l'on a par trop cherché à tout vouloir expliquer par le $p H$ alors qu'il ne s'agit là que d'une résul. tante dépendant d'une foule de facteurs ; qu'en particulier, comme l'a rappelé $M$. Lefèvae (1), le degré hydrotimétrique d'une eau n'est nullement proportionnel au $p H$ : "Si le complexe physicochimictue dit-il, possède une grande marge de stabilité (effet tampon), les conséquences chimiques du métabolisme du peuplement se feront peu ou pas sentir. le $p H$ restera stable. Si, au contraire, le complexe physicochimique est en équilibre instable, l'action biochimique du peuplement amènera une variation du $p H$ entre des limites impossibles à prévoir."

(1) Signiflcation et valeur du facteur $p H$ en Hydrobiologie. - Bulletin de la Société Centrale d'Aquiculture et de Péche. no 7-12, Juillet-Décembre 1940, p. 50. 
Et il en Tonclut " le pH envisagé seul n'a donc dans la plupart des cas, qu'une signification bien minime en Hydrobiologie ".

Mais si, d'une façon générale, nous sommes entièrement d'accord sur cette conclusion, il faut bien noter que les $p H$ sur lesquels a porté l'étude de M. Lefévre sont des $p H$ moyens ou assez voisins de la moyenne; ils sont exactement compris entre 5,7 (la Bonne Mare en Forêt de Rambouillet et 8,6 (étang du Rondeau, Parc de Rambouillet) et nous pensons qu'il conviendrait de ne pas généraliser, et, en particulier, de ne pas étendre les conclusions relatives à la non signification des $p H$ en Hydrobịologie, à ses très grandes ou à ses très petites valeurs, ce qui constituerait, semble-t-il, une extrapolation dangereuse.

Certes le $\mathrm{pH}$ ne saurait jamais être une cause ; ce n'est qu'une résultante ; mais s'il est bien certain que les valeurs moyennes dur $p H$ oscillent, en un même point, entre des valeurs assez diverses et de ce fait ne présentent guère d'intérêt en aquiculture, les très grosses ou les très petites semblent cependant avoir une signification.

C'est ainsi que Schaperciaus, dans un ourrage aujourd'hui classique (1), et bien qu'ayant insisté dès cette époque (1933) sur ce qu'il est faux de supposer que les eaux à forte teneur en chaux possèdent un $p H$ de valeur élevée et doivent donc être particulièrement alcalines, et sur ce que le contraire est plutôt le cas, a montré qu'il existait pour le $p H$ deux points au-dessus et au-dessous desquels il y avait danger pour le Poisson et qu'il appelle d'ailleurs " points dangereux " (Gefahrpunkt) : ce sont les valeurs 4,8 et 9 .

Selon les constatations de Scraperclaus, les Poissons, dans une eau à $p H$ de 5 et au-dessous, sont atteints de maladies de la peau et des branchies et finalement en meurent. Ie point d'acidité dangereux (säurer Gefahrpunkt) serait pour la Carpe de 4,8. Un tel $p H$ agirait mortellement sur le Poisson en un temps très court. Ce point dangereux cependant, qui dépend de plusieurs facteurs, peut varier sensiblement : en présence de la glace par exemple, il serait plus élevé. La sensibilité des autres poissons d'étang aux très faibles valeurs du $\mathrm{pH}$ serait tout à fait analogue.

De même, il existe un point dangereux supérieur (alkalischer Gefahrpunkt) qui serait de 9. Ce point peut être toutefois dépassé dans la journée, notamment dans l'après-midi, sur les bords des étangs là où, pour l'assimilation chlorophyllienne, la végétation extrait le gaz carbonique du bicarbonate de chaux pour ne laisser subsister que la chaux, mais alors les Poissong s'échappent dans les eaux profondes jusqu'à ce que, dans la nuit, les conditions normales soient à nouveau revenues.

C'est peut-être ce qui se produit dans certains étangs de Courville, relativement profonds, où nous avons parfois constaté sur les bords, des $p H$ de valeurs élevées $(9,2$ en Juin dans l'étan̊ $4 ; 9.2$ dans l'étang 7 en Juillet).

(1) Lehrbuch der Teichwirtschaft. - Berlin, Paul PAREY, 1933. 
Nous pensons donc que, dans une eau peu profonde, où le $p H$ atteint la valeur de 11 au début de l'été (cas des rivières anglaises du Domaine national de Rambouillet), la Carpe se trouve placée dans de mauvaises conditions préjudiciables à sa fraye.

Si donc l'absence de végétation phanérogamique (canaux) ou la déficience de ladite végélation (Rondeau) pouvait être la cause principale ou, tout au moins, l'une des causes de ce que la Carpe ne frave pas et ne fraye que peu dans ces plans d'eau, le fait que le même phénomène se constate dans les rivières anglaises abondamment pourvues de végétation ne va pas forcément à l'encontre de la précédente lyppothèse, parce que les rivières anglaises ne sont pas strictement comparables aux canaux et au Rondeau : il pourrait se faire, en effet, que dans les rivières anglaises de Rambouillet l'absence de la fraye de la Carpe soit imputable à une toute autre cause manifestée par l'existence d'un $p H$ anormalement ilevé et de valeur supérieure, au début de l'été, à l'atlkalischer Géfarhrpunkt de Schaperclaus.

Nous n'avons certes pas la prétention d'avoir ainsi établi la vérité et nous ne considérons ce qui précède que comme une simple remarque : nous pensons mềme que l'action directe du tannin sur la fraye de la Carpe est peut-être réelle ! Ce que nous avons voulu simplement démantrer, c'est que le phénomène de la fraye de la Carpe, dans les canaux du Domaine national de Rambouillet (ou ailleurs), n'est peut-être pas aussi simple qu'on pourait le croire tout d'abord; de mème en ce qui concerne l'influence des très grosses ou des très petites valeurs du $p H$ : les facteurs à intervenir sont multiples et l'on ne saurait en dénouer la trame qu'avec une extrếme prudence.

\section{pourcentage D'Aleving produts, pan espèces, DANS I.Fs Étangs D'ALEviNage}

Les Sociétés de péche demandent surtout du Rotengle, puis de la Tanche et après, mais en bien moins grande quantite, de la Carpe. L'aménagement piscicole de Courville répond à ces désirs.

L'empoissonnement moyen à l'hectare est, ainsi que nous l'avons déjà dit, de 7 Carpes (3 femelles, 4 males), 25 Tanches (dont 12 femelles et 13 males) et 150 Rotengles (de sexe indéterminé).

Si l'on veut se rendre compte de I'influence, sur la production, des seules conditions naturelles, il convient de laisser de côté ce qui a trait aux alevins de deux étés puisqu'il y a eu pour eux un choix “volontaire du nombre d'alevins mis en réserve de lelles ou telles espèces, parmi lesquelles la Carpe, par exemple, n'est jamais comprise.

Nous n'examinerons donc ici que ce qui concerne les alevins d'un été.

De 1938 à 1942, soit en cinq années, il en a été produit à Courville 1.586.690, la surface en eau consacrée à ces alevins étant en moyenne de 
6 h. 30 a. Mais, en fait, de ces cinq années, seules 1940 et 1942 peuvent être réellement comparées, car c'est seulement en 1940 et 1942 que les surfaces en eau consacrées aux alevins d'un été étaient identiques. Dans ces deux années, la production annuelle moyenne a été, au total, de 260.461 alevins d'un été, correspondant à une production moyenne annuelle à l'hectare de 41.343 alevins.

Cette production, du moins de 1939 à 1942 , se décompose comme suit :

\begin{tabular}{|c|c|c|c|c|c|c|c|}
\hline \multirow[b]{2}{*}{ Annees } & \multirow{2}{*}{$\begin{array}{l}\text { Produc- } \\
\text { tion totale } \\
\text { en alevins } \\
\text { d'un eté }\end{array}$} & \multicolumn{2}{|c|}{$\begin{array}{l}\text { Production en gardons } \\
\text { d'un eté }\end{array}$} & \multicolumn{2}{|c|}{$\begin{array}{c}\text { Praduction en tanches } \\
\text { d'un ele }\end{array}$} & \multicolumn{2}{|c|}{$\begin{array}{l}\text { Production en alevins } \\
\text { d.-Carpes d'un eté }\end{array}$} \\
\hline & & Nombre & $\begin{array}{l}\text { Pourcen- } \\
\text { tage par } \\
\text { rapport a la } \\
\text { produc- } \\
\text { tion totale }\end{array}$ & Nombre & $\begin{array}{c}\text { Pourcen- } \\
\text { 1age par } \\
\text { rapport a la } \\
\text { produc- } \\
\text { tion to tale }\end{array}$ & Nombre & $\begin{array}{c}\text { Pourcen- } \\
\text { tape par } \\
\text { rapport a la } \\
\text { produc } \\
\text { tíon totale }\end{array}$ \\
\hline $1939 \ldots \ldots$ & 309.488 & 247.750 & $80 \%$ & 44.000 & $14,3 \%$ & 17.738 & $5,7 \%$ \\
\hline $1940 \ldots \ldots$ & 303.700 & 292,000 & $96,2 \%$ & 7.938 & $42,6 \%$ & 3.762 & $1,2 \%$ \\
\hline $1941 \ldots \ldots$ & 203.397 & 165.894 & $82 \%$ & 30.680 & $15 \%$ & 6.823 & $3 \%$ \\
\hline $1942 \ldots \ldots$ & 217.223 & 171.132 & $78,8 \%$ & 23.463 & $10,8 \%$ & 22.628 & $10,4 \%$ \\
\hline Moyennes. & 258.452 & 219.194 & $84,3 \%$ & 26.520 & $10,7 \%$ & 12.738 & $5 \%$ \\
\hline
\end{tabular}

Dans les conditions de notre alevinage, la proportion des Gardons est donc toujours énorme par rapport à celle des Tanches et des Carpes : $84,3 \%$ de la production totale des alevins d'un été, - contre 10,7\% pour les Tanches et $5^{1} \%$ pour les Carpes.

Au fait, les moyennes ne sont trop souvent que de simples constructions de l'esprit, aussi est-il plus intéressant d'examiner lés variations des chiffres de production, plutót que leur moyenne.

On constate ainsi que l'alevinage des Gardons paraît assez régulier, qu'il oscille peu autour du chiffre moyen. Si les chiffres de 1941 et 1942 sont inférieurs à ceux de 1939 et 1940 , nous sommes portés à croire, sans en avoir la certitude, que le fait en est peut-être bien imputable on granđe partie, tout simplement, aux dépradations des Canards.

Quant à la Tanche, si l'on excepte l'année 1940, très anormale en ce qui concerne cette espèce, son alevinage a été en somme, très régulier : — 44.000 alevins en $1939,-30.680$ en 1941 , — 23.463 en 1942 .

Pour la Carpe, au contraire, il n'y a aucune ronstance, mais des sautes brusques d'une année à l'autre : -17.738 alevins en $1939,-3.762$ seulement en 1940 , - 6.823 en 1941 , - 22.628 en 1942 ; nous avons l'intention d'examiner ce phénomène de très près et d'en rechercher les causes. 
Le fait est peut-être bien en rapport avec les variations des conditions atmosphériques. C'est qu'en efiet, ainsi que nous l'avons déjà dit, la Carpe paraît frayer dès que la température de l'eau atteint 19 à $20^{\circ}$. Or si, dans la suite, des froids interviennent, une grande partie du frai de Carpe est perdue. C'est la raison pour laquelle, nous réservons chaque année en eau froide, un certain nombre de Carpes pour ne les déverser en étang où les faire poser dans les frayères "Dubish "que vers le $10 \mathrm{Juin}$ environ, afin de parer à la déficience éventuelle des premières frayes. Nous serions d'ailleurs portés à croire qu'il conviendrait de ne déverser aucun géniteur Carpe en étang en Avril et Mai, mais de les maintenir tous en eau froide, pour ne les mettre en place dans les étangs de pose qu'au début de Juin, afin d'éviter la période durant laquelle peuvent se produire des abaissements de température préjudiciables a la fraye de cette espèce. Des expériences sont actuellement en cours à ce sujet. 